\title{
One-pot, three-component Synthesis of pyrrolo[2,3-d]pyrimidine Derivatives
}

\author{
Ramin Javahershenas, Jabbar Khalafy* \\ Department of Organic Chemistry, Faculty of Chemistry, Urmia University, Urmia, Iran. \\ * Corresponding author's e-mail: jkhalafi@yahoo.com; j.khalafi@urmia.ac.ir Tel/Fax: +98-44-3275-5294
}

Received October 25 ${ }^{\text {th }}, 2017$; Accepted February $7^{\text {th }}, 2018$.

DOI: http://dx.doi.org/10.29356/jmcs.v62i1.340

\begin{abstract}
A green approach for the synthesis of polyfunctionalized pyrrolo[2,3-d]pyrimidine derivatives was successfully achieved by a one-pot three-component reaction of arylglyoxals, 6-amino-1,3-dimethyluracil and barbituric acid derivatives in the presence of tetra- $n$-butylammonium bromide (TBAB) $(5 \mathrm{~mol} \%)$ as the catalyst in ethanol at 50 ${ }^{\circ} \mathrm{C}$. This protocol has many advantages such as high yields (73-95\%), green and simple procedure, short reaction times, easy work-up, mild reaction conditions and general applicability.

Key words: 6-Amino-1,3-dimethyluracil; arylglyoxals; barbituric acid derivatives; TBAB; pyrrolo[2,3-d]pyrimidines; one-pot; multicomponent reactions.

Resumen. Se describe el desarrollo de una ruta sintética sustentable para la preparación de derivados pirrolo[2,3d]pirimidinas mediante una reacción de tres componentes en un matraz de arilglioxales, 6-amino-1,3-dimethyluracilo y derivados del ácido barbitúrico en presencia de bromuro de tetra- $n$-butilamonio (TBAB) (5\% mol) como catalizador en etanol a $50{ }^{\circ} \mathrm{C}$. Este protocolo posee diversas ventajas, tales como elevados rendimientos (73-95\%), procedimiento simple y sustentable, cortos tiempos de reacción, extracción sencilla, condiciones de reacción suaves y amplia aplicabilidad.
\end{abstract}

Palabras clave: 6-Amino-1,3-dimetiluracilo; arilglioxales; derivados del ácido barbitúrico; TBAB; pirrolo[2,3d]pirimidinas; reacción multicomponente en un matraz.

\section{Introduction}

There is a worldwide demand for design and synthesis of organic compounds with biological and pharmaceutical activity [1] using readily available starting materials by one-pot multicomponent reactions (MCRs) [2]. MCRs have many advantages in comparison with classical reactions, such as easier isolation and purification, lower energy consumption, using green solvents, easy operation, and more productivity with excellent chemo- and regioselectivities [3].

Barbituric acid and uracil derivatives have an important role in medicinal chemistry because for their biological activities [4]. There are many multicomponent condensations using barbituric acid and uracil as starting materials for the rapid synthesis of heterocyclic of pyrido-, pyrrolo-, and pyrimido-pyrimidines with various biological and pharmaceutical application [5]. Examples of their use are as antimicrobial [6], acaricidal [7], anti-inflammatory [8], anticancer [9], analgesic [10], sedative [11], anticonvulsants [12] and anesthetic agents [13].

Recently tetra- $n$-butylammonium bromide (TBAB) has emerged as one of the most widely used phase transfer catalysts. It combines the lipophilicity required for an efficient phase transfer catalyst with the hydrophobicity necessary for efficient catalyst recovery [14]. This and some other related ionic liquids have considerable interest as potential ecofriendly reagents due to their low vapor pressure. These liquids dissolve in many inorganic and organic materials, are nonvolatile and nonflammable, possess high thermal, high ionic conductivity, and chemical stability. It has been successfully used in the solid-liquid or liquid-liquid phase-transfer alkylation for $\mathrm{C}-\mathrm{C}$ and $\mathrm{C}-\mathrm{X}$ bond formation. It has been used as an inexpensive, mild, water-tolerant, and environmentally compatible an efficient homogeneous catalyst in different organic transformations [15]. TBAB is also a cheap, simple to use, readily available ionic liquid and has inherent properties like non-corrosive nature environmental compatibility, and ease of reusability [16].

In continuation of our interest in the synthesis of new heterocyclic compounds via one-pot, multicomponent reactions [17-23], herein we report a convenient and rapid method for the synthesis of pyrrolo[2,3-d]pyrimidine 
derivatives by one-pot three-component reactions using arylglyoxals 1a-d, 6-amino-1,3-dimethyluracil (2) and barbituric acid derivatives 3a-c in the presence of a catalytic amount of TBAB (5 mol\%) at $50{ }^{\circ} \mathrm{C}$ in ethanol.

\section{Results and Discussion}

In attempting to develop a simple, one-pot and short reaction pathway for the synthesis of various heterocyclic compounds, we reported earlier the synthesis of pyrazolo[3,4- $d$ ]pyridines, pyrazolo[3,4- $b$ ]qunolin-5-ones and pyrazolo[4',3':5,6]pyrido[2,3-d]pyridin-5,7-diones, benzo[H]thieno[2,3-b]quinolone-9-yl(aryl)methanones, 6,7-dihydro$1 H$-indole-4(5H)-ones, acridine-1,8(2H,5H)-diones, 1,3-dimethyl-2,4-dioxo-1,2,3,4,5,8-hexahydropyrido[2,3d]pyrimidine-6-carbonitriles, pyrrolo[3,2-d]pyrimidines [17-23].

The reaction of arylglyoxal 1a with $\mathbf{2}$, and barbituric acid derivatives $\mathbf{3 a}$ and $\mathbf{3 c}$ in ethanol under reflux conditions to form the desired polyfunctionalized pyrrolo[2,3- $d]$ pyrimidine derivatives $\mathbf{4 e}$ and $\mathbf{4 i}$ has been previously reported [24]. Herein we report a simpler and more efficient procedure for this reaction.

We found that the reaction of arylglyoxals 1a-d with $\mathbf{2}$ and barbituric acid derivatives 3a-c in the presence of TBAB (5 mol\%) in ethanol at $50{ }^{\circ} \mathrm{C}$, instead of using reflux conditions, afforded polyfunctionalized pyrrolo[2,3d]pyrimidine derivatives $\mathbf{4 a - 1}$ in high yields with no sign of any dihydropyrido[2,3-d:6,5-d']dipyrimidine derivatives 5aI formation (Scheme 1).

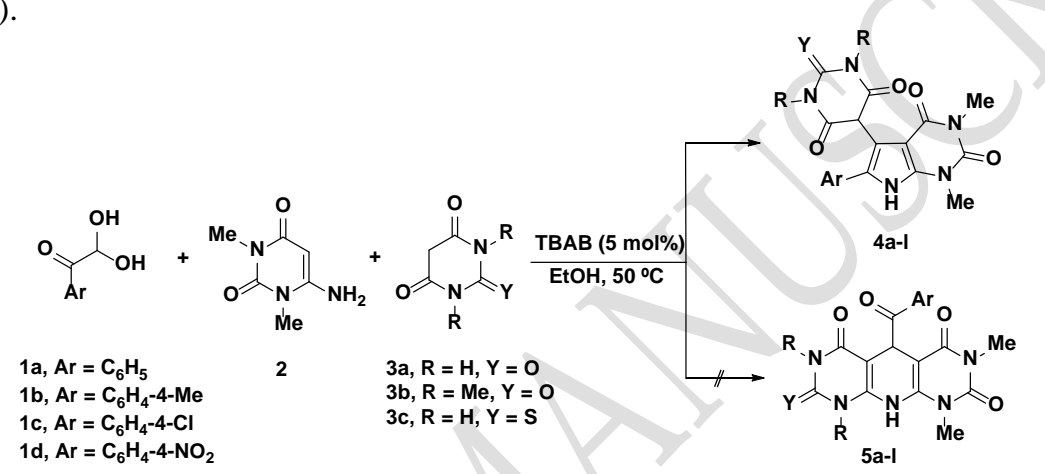

Scheme 1. Synthesis of pyrrolo[3,2-d]pyrimidine derivatives 4a-l.

In our trial studies, the reaction of phenylglyoxal 1a, 2, and barbituric acid 3a was chosen as a model reaction (Table 1). First, we carried out this model reaction in the absence of a catalyst, but no product was observed even after $30 \mathrm{~min}$ of stirring at room temperature (Table 1, entry 1). To optimize the reaction temperature, the reactions were carried out at different temperatures ranging from room temperature to reflux. It was found that the yield was improved when the temperature was increased to reflux. Refluxing the reaction mixture in EtOH without any catalyst for 30 min gave the desired product $4 \mathrm{a}$ in $81 \%$ yield (Table 1, entry 3). Using 5 mol\% of TBAB as organocatalyst for this reaction improved the yield (Table 1, entry 6), but using $10 \mathrm{~mol} \%$ of TBAB for 65 min decreased the yield to 78\% (Table 2, entry 9). The product was fully characterized using FT-IR (KBr), ${ }^{1} \mathrm{H}-\mathrm{NMR}$ and ${ }^{13} \mathrm{C}-\mathrm{NMR}$ spectral data, and by reference to the physical data, where appropriate [24].

To optimize the reaction temperature, the reactions were carried out at different temperatures ranging from room temperature to reflux. It was found that the yield was improved and the reaction time was shortened when the temperature was increased to $50{ }^{\circ} \mathrm{C}$.

The best result was obtained in terms of yield (90\%) and reaction time (65 min) when the reaction was performed using $5 \mathrm{~mol} \%$ of TBAB (Table 1, entry 6). To find the best solvent for this reaction, we repeated the model reaction in various solvents such as acetonitrile, dichloromethane (DCM), THF, DMF, $\mathrm{MeOH}, \mathrm{EtOH} / \mathrm{H}_{2} \mathrm{O}(1: 1)$ and $\mathrm{H}_{2} \mathrm{O}$. It was shown that EtOH was the best solvent in terms of yield and reaction time (Table 1, entry 6). 
Table 1. The effect of several solvents, temperatures and mol\% of TBAB in the synthesis of $\mathbf{4 a}$

\begin{tabular}{|c|c|c|c|c|c|}
\hline Entry & Catalyst & Temperature $\left({ }^{\circ} \mathrm{C}\right)$ & Solvent & Time (min) & Yield (\%) \\
\hline 1 & No Catalyst & RT & $\mathrm{EtOH}$ & 30 & trace \\
\hline 2 & No Catalyst & 50 & $\mathrm{EtOH}$ & 30 & 56 \\
\hline 3 & No Catalyst & Reflux & $\mathrm{EtOH}$ & 30 & 81 \\
\hline 4 & TBAB (5 mol\%) & $\mathrm{RT}$ & $\mathrm{EtOH}$ & 65 & 38 \\
\hline 5 & TBAB (5 mol\%) & 40 & $\mathrm{EtOH}$ & 65 & 65 \\
\hline 6 & TBAB (5 mol \%) & 50 & EtOH & 65 & 90 \\
\hline 7 & ТВАВ (5 mol\%) & 60 & $\mathrm{EtOH}$ & 65 & 89 \\
\hline 8 & TBAB (5 mol\%) & Reflux & $\mathrm{EtOH}$ & 65 & 85 \\
\hline 9 & TBAB (5 mol\%) & 50 & THF & 65 & 36 \\
\hline 10 & TBAB (5 mol\%) & 50 & $\mathrm{MeOH}$ & 65 & 54 \\
\hline 11 & TBAB (5 mol\%) & 50 & $\mathrm{CH}_{2} \mathrm{Cl}_{2}$ & 65 & 25 \\
\hline 12 & TBAB (5 mol\%) & 50 & $\mathrm{CH}_{3} \mathrm{CN}$ & 65 & 31 \\
\hline 13 & TBAB (5 mol\%) & 50 & $\mathrm{DMF}$ & 65 & 51 \\
\hline 14 & TBAB (5 mol\%) & 50 & $\mathrm{H}_{2} \mathrm{O}$ & 65 & 56 \\
\hline 15 & TBAB (5 mol \%) & 50 & $\mathrm{EtOH} / \mathrm{H}_{2} \mathrm{O}(1: 1)$ & 65 & 61 \\
\hline
\end{tabular}

We also examined this reaction in the absence and presence of several other catalysts. When the reaction was carried out without any catalyst, the desired product $\mathbf{4 a}$ was formed in 56\% yield (Table 2, entry 1). The use of bases like $\mathrm{K}_{2} \mathrm{CO}_{3}$ or 1,4-diazabicyclo[2.2.2] octane (DABCO) led to no improvement in yield, while acids, $p$-toluene sulfonic acid ( $p$-TSA), $\mathrm{ZrOCl}_{2}$, sodium dodecyl sulfate (SDS), L-Proline gave small improvements (Table 2, entries 2-7). The best result was obtained when TBAB was used as a catalyst giving a yield of $90 \%$ (Table 2, entry 8).

To study the effect of the amount of catalyst, the reactions were carried out using different amounts of TBAB ranging from 5 to $10 \mathrm{~mol} \%$. The use of $5 \mathrm{~mol} \%$ TBAB in EtOH led to optimum results. Using the larger amounts of TBAB did not improve the yields.

Table 2. The effect of several catalysts in the synthesis of $\mathbf{4 a}{ }^{a}$

\begin{tabular}{cccccc}
\hline Entry & Catalyst & Temperature $\left({ }^{\circ} \mathrm{C}\right)$ & Solvent & Time (min) & Yield (\%) \\
\hline 1 & - & 50 & EtOH & 65 & 56 \\
2 & $\mathrm{~K}_{2} \mathrm{CO}_{3}(5 \mathrm{~mol} \%)$ & 50 & EtOH & 65 & 49 \\
3 & $\mathrm{DABCO}^{(5 \mathrm{~mol} \%)}$ & 50 & EtOH & 65 & 45 \\
4 & $\mathrm{SDS}(5 \mathrm{~mol} \%)$ & 50 & EtOH & 65 & 65 \\
5 & p-TSA (5 mol\%) & 50 & EtOH & 65 & 67 \\
6 & ZrOCl $_{2}(5 \mathrm{~mol} \%)$ & 50 & EtOH & 65 & 69 \\
7 & L-Proline (5 mol\%) & 50 & EtOH & 65 & 71 \\
$\mathbf{8}$ & TBAB (5 mol\%) & 50 & EtOH & $\mathbf{6 5}$ & $\mathbf{9 0}$ \\
9 & TBAB (10 mol\%) & 50 & EtOH & 65 & 78 \\
\hline
\end{tabular}

a Phenyglyoxal (1 mmol), barbituric acid (1 mmol) and 2 (1 mmol) in EtOH (5 mL).

The reaction times (60-80 min), yields (73-95\%) and melting points $\left(>300{ }^{\circ} \mathrm{C}\right)$ of synthesized pyrrolo[3,2d]pyrimidine derivatives 4a-l using 5 mole\% TBAB as catalyst are shown in Table 3. The substituted pyrrolo[3,2d]pyrimidine derivatives 4a-I were characterized using FT-IR, ${ }^{1} \mathrm{H}$ NMR and ${ }^{13} \mathrm{C}$ NMR spectral data and microanalysis. In the ${ }^{1} \mathrm{H}$ NMR spectra of products $4 \mathbf{a}-\mathbf{l}$, the characteristic singlets at around 11.79-12.29 and 4.55-5.32 ppm are attributed to the $\mathrm{NH}$ of pyrrole moiety and the $\mathrm{CH}$ of barbituric acid ring respectively. In the ${ }^{13} \mathrm{C}$ NMR spectra of the products 4a-l, signals located around 151.1-174.2 ppm were ascribed to the three different carbonyl groups. In the FTIR $(\mathrm{KBr})$ spectra, the characteristic absorptions bands at 1671-1703, 1634-1689 and 1549-1589 $\mathrm{cm}^{-1}$ could be assigned to the vibrations of different carbonyl groups. 
Table 3. Reaction conditions for the synthesis of pyrrolo[3,2-d]pyrimidine $4 \mathbf{4 a - 1}$ a $^{\text {a }}$

\begin{tabular}{cccccccc}
\hline Product & $\mathrm{Ar}$ & $\mathrm{R}$ & $\mathrm{R}$ & $\mathrm{Y}$ & $\begin{array}{c}\text { Time } \\
(\mathrm{min})\end{array}$ & Yields $(\%)$ & $\mathrm{Mp}\left({ }^{\circ} \mathrm{C}\right)$ \\
\hline $\mathbf{4 a}$ & $\mathrm{C}_{6} \mathrm{H}_{5}$ & $\mathrm{O}$ & $\mathrm{H}$ & $\mathrm{H}$ & 65 & 90 & $>300$ \\
$\mathbf{4 b}$ & $4-\mathrm{MeC}_{6} \mathrm{H}_{4}$ & $\mathrm{O}$ & $\mathrm{H}$ & $\mathrm{H}$ & 60 & 95 & $>300$ \\
$\mathbf{4 c}$ & $4-\mathrm{ClC}_{6} \mathrm{H}_{4}$ & $\mathrm{O}$ & $\mathrm{H}$ & $\mathrm{H}$ & 70 & 78 & $>300$ \\
$\mathbf{4 d}$ & $4-\mathrm{NO}_{2} \mathrm{C}_{6} \mathrm{H}_{4}$ & $\mathrm{O}$ & $\mathrm{H}$ & $\mathrm{H}$ & 75 & 76 & $>300$ \\
$\mathbf{4 e}$ & $\mathrm{C}_{6} \mathrm{H}_{5}$ & $\mathrm{~S}$ & $\mathrm{H}$ & $\mathrm{H}$ & 70 & 86 & $>300$ \\
$\mathbf{4 f}$ & $4-\mathrm{MeC}_{6} \mathrm{H}_{4}$ & $\mathrm{~S}$ & $\mathrm{H}$ & $\mathrm{H}$ & 65 & 90 & $>300$ \\
$\mathbf{4 g}$ & $4-\mathrm{ClC}_{6} \mathrm{H}_{4}$ & $\mathrm{~S}$ & $\mathrm{H}$ & $\mathrm{H}$ & 75 & 76 & $>300$ \\
$\mathbf{4 h}$ & $4-\mathrm{NO}_{2} \mathrm{C}_{6} \mathrm{H}_{4}$ & $\mathrm{~S}$ & $\mathrm{H}$ & $\mathrm{H}$ & 80 & 73 & $>300$ \\
$\mathbf{4 i}$ & $\mathrm{C}_{6} \mathrm{H}_{5}$ & $\mathrm{O}$ & $\mathrm{Me}$ & $\mathrm{Me}$ & 60 & 91 & $>300$ \\
$\mathbf{4 j}$ & $4-\mathrm{MeC}_{6} \mathrm{H}_{4}$ & $\mathrm{O}$ & $\mathrm{Me}$ & $\mathrm{Me}$ & 55 & 94 & $>300$ \\
$\mathbf{4 k}$ & $4-\mathrm{ClC}_{6} \mathrm{H}_{4}$ & $\mathrm{O}$ & $\mathrm{Me}$ & $\mathrm{Me}$ & 65 & 82 & $>300$ \\
$\mathbf{4 l}$ & $4-\mathrm{NO}_{2} \mathrm{C}_{6} \mathrm{H}_{4}$ & $\mathrm{O}$ & $\mathrm{Me}$ & $\mathrm{Me}$ & 70 & 75 & $>300$ \\
\hline
\end{tabular}

a Phenyglyoxal (1 mmol), barbituric acid (1 mmol) and 2 (1 mmol) in EtOH (5 mL), $50{ }^{\circ} \mathrm{C}, 65 \mathrm{~min}$.

A plausible mechanism for the formation of the products $\mathbf{4 a - 1}$ is shown in Scheme 2. A sequence of reactions involving Knoevenagel condensation of $\mathbf{2}$ with aryglyoxals in the presence of TBAB forms the corresponding intermediate $\mathbf{I}$. The observed products were then formed through an intermolecular condensation of intermediate $\mathbf{I}$ with barbituric acid derivatives with the ketone carbonyl group of $\mathbf{I}$ and loss of a water molecule, as shown in Scheme 2 . It is expected that the use of TBAB in ethanol increases the stability of the ionized or tautomeric structures, thereby increasing the reaction rate and yields. 


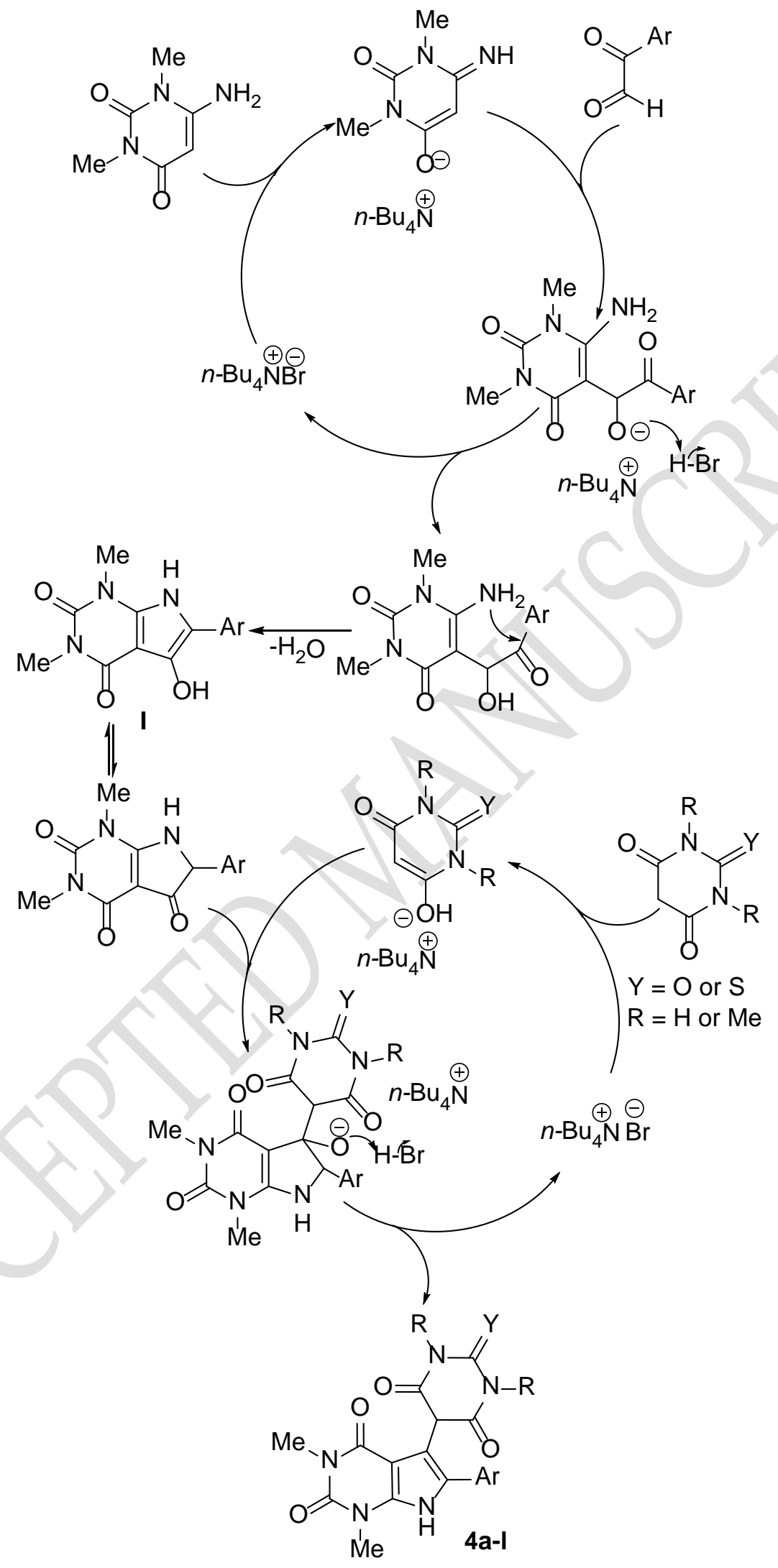

Scheme 2. The proposed mechanism for the one-pot, three-component reaction for the preparation of derivatives $\mathbf{4 a - 1}$. 


\section{Conclusion}

We have synthesized a new series of pyrrolo[2,3-d]pyrimidine derivatives 4a-I in high yields, by the one-pot threecomponent reaction of arylglyoxals 1a-d with 1,3-dimethyl-6-aminouracil 2 and barbituric acid derivatives 3a-c using 5 mol\% TBAB as the catalyst in ethanol at $50{ }^{\circ} \mathrm{C}$. The simplicity of the method, ease of product isolation, mild reaction conditions, high yields, short reaction times and availability of the starting materials are the advantages of this procedure. Our method provides a simple synthesis of polyfunctionalized pyrrolo[2,3- $d$ ]pyrimidine derivatives with different substituents, which may have pharmaceutical and biological applications.

\section{Experimental}

The chemicals used in this work were purchased from Acros and Merck companies and were used without purification. Freshly distilled solvents were used throughout and anhydrous solvents were dried according to Perrin and Armarego [25]. Melting points were measured on an Electrothermal 9200 apparatus and are uncorrected. FT-IR (KBR) spectra were recorded on a Thermo Nicolet (Nexus 670) spectrometer using $\mathrm{KBr}$ discs. ${ }^{1} \mathrm{H}(300 \mathrm{MHz})$ and ${ }^{13} \mathrm{C}(75.5$ $\mathrm{MHz}$ ) NMR spectra were recorded on a Bruker DRX-300 Avance spectrometer in DMSO- $d_{6}$ with TMS as the internal reference. Elemental analyses were performed using a Leco Analyzer 932. The arylglyoxals were prepared as their hydrates by oxidation of the corresponding acetophenones with $\mathrm{SeO}_{2}$ [22].

\section{General procedure for synthesis of pyrrolo[2,3-d]pyrimidine derivatives}

A mixture of arylglyoxals 1a-d (1 mmol), 1,3-dimethyl-6-aminouracil (2) (155 mg, $1 \mathrm{mmol}$ ) and barbituric acid derivatives 3a-c $(1 \mathrm{mmol})$, TBAB $(16 \mathrm{mg}, 0.05 \mathrm{mmol})$ in ethanol $(5 \mathrm{ml})$ was stirred at $50{ }^{\circ} \mathrm{C}$ for the appropriate time as indicated in Table 3. The reaction was monitored by thin-layer chromatography TLC using (EtOAc/hexane, 5:1) as eluent. After completion of the reaction, the solid precipitate was filtered, washed with cold water and dried. Recrystallization from ethanol gave the desired products in 73-95\% yields in Table 3.

5-(6-(Phenyl)-1,3-dimethyl-2,4-dioxo-2,3,4,7-tetrahydro-1H-pyrrolo[2,3-d]pyrimidin-5-yl)pyrimidine-

2,4,6(1H,3H,5H)-trione (4a). White powder; $343 \mathrm{mg}$; $\mathrm{mp}>300{ }^{\circ} \mathrm{C}$; FT-IR (KBr) $v_{\max } 3468,3173,3066,2954,1671$, 1655, 1563, 1444, 1394, 1305, 1097, 1114, 1021, 979, 842, $748 \mathrm{~cm}^{-1} ;{ }^{1} \mathrm{H}$ NMR (DMSO-d, $\left.300 \mathrm{MHz}\right) \delta 11.85(1 \mathrm{H}, \mathrm{s}$, exchanged by $\mathrm{D}_{2} \mathrm{O}$ addition, $\left.\mathrm{NH}\right), 11.17$ (2H, s, exchanged by $\mathrm{D}_{2} \mathrm{O}$ addition, $\left.2 \times \mathrm{NH}\right), 7.55(2 \mathrm{H}, \mathrm{d}, J=6.9 \mathrm{~Hz}, \mathrm{Ar}), 7.48$ (2H, t, $J=7.2 \mathrm{~Hz}, \mathrm{Ar}), 7.39$ (1H, d, $J=6.6 \mathrm{~Hz}, \mathrm{Ar}), 5.00$ (1H, s, CH), 3.50 (3H, s, 3-Me), 3.15 (3H, s, 1-Me); ${ }^{13} \mathrm{C} \mathrm{NMR}$ (DMSO-d, $75.5 \mathrm{MHz}) \delta 169.7$ (C=O carbonyl), 159.1 (C=O carbonyl), 151.1 (C=O carbonyl), 139.7, 131.9, 130.9, 130.1, 129.7, 129.2 and 128.5 (aromatic C), 108.9 and 97.5 (pyrrole C), $48.1(\mathrm{CH}), 29.8$ and 27.7 ( $\left.\mathrm{CH}_{3}\right)$. Anal. Calcd for $\mathrm{C}_{18} \mathrm{H}_{15} \mathrm{~N}_{5} \mathrm{O}_{5}$ : C, 56.69; H, 3.96; N, 18.37. Found: C, 56.76; H, 4.11; N, 18.30.

5-(6-(4-Methylphenyl)-1,3-dimethyl-2,4-dioxo-2,3,4,7-tetrahydro-1H-pyrrolo[2,3-d]pyrimidin-5-yl)pyrimidine2,4,6(1H,3H,5H)-trione (4b). White powder; $375 \mathrm{mg}$; mp >300 ${ }^{\circ} \mathrm{C}$; FT-IR (KBr) $v_{\max } 3410,3226,3114,2958,1702$, 1652, 1561, 1443, 1357, 1241, 1041, 974, $818 \mathrm{~cm}^{-1}$; ${ }^{1} \mathrm{H}$ NMR (DMSO-d $\left.{ }_{6}, 300 \mathrm{MHz}\right) \delta 11.79\left(1 \mathrm{H}\right.$, s, exchanged by $\mathrm{D}_{2} \mathrm{O}$ addition, $\mathrm{NH}), 11.16\left(2 \mathrm{H}, \mathrm{s}\right.$, exchanged by $\mathrm{D}_{2} \mathrm{O}$ addition, $\left.2 \times \mathrm{NH}\right), 7.42(2 \mathrm{H}, \mathrm{bs}, \mathrm{Ar}), 7.29(2 \mathrm{H}, \mathrm{bs}, \mathrm{Ar}), 4.96(1 \mathrm{H}, \mathrm{s}, \mathrm{CH})$, 3.47 (3H, s, 3-Me), 3.15 (3H, s, 1-Me), 2.34 (3H, s, Me); ${ }^{13} \mathrm{C}$ NMR (DMSO-d, $\left.75.5 \mathrm{MHz}\right) \delta 169.7$ (C=O carbonyl), 159.1 (C=O carbonyl), 151.0 ( $\mathrm{C}=\mathrm{O}$ carbonyl), 139.5, 131.9, 130.3, 129.8, 129.7, 129.0 and 128.4 (aromatic C), 108.5 and 97.4 (pyrrole C), $47.0(\mathrm{CH}), 31.1,28.9$ and $21.2\left(\mathrm{CH}_{3}\right)$. Anal. Calcd for $\mathrm{C}_{19} \mathrm{H}_{17} \mathrm{~N}_{5} \mathrm{O}_{5}$ : C, 57.72; H, 4.33; N, 17.71 . Found: C, 57.79; H, 4.25; N, 17.81

5-(6-(4-Chlorophenyl)-1,3-dimethyl-2,4-dioxo-2,3,4,7-tetrahydro-1H-pyrrolo[2,3-d]pyrimidin-5-yl)pyrimidine2,4,6(1H,3H,5H)-trione (4c). Pale yellow powder; $324 \mathrm{mg} ; \mathrm{mp}>300{ }^{\circ} \mathrm{C}$; FT-IR (KBr) $v_{\max } 3412,3209,2955,1702$, $1655,1564,1495,1438,1361,1248,1097,1031,977,809,505 \mathrm{~cm}^{-1}$; ${ }^{1} \mathrm{H}$ NMR (DMSO- $\left.d_{6}, 300 \mathrm{MHz}\right) \delta 11.91(1 \mathrm{H}, \mathrm{s}$, exchanged by $\mathrm{D}_{2} \mathrm{O}$ addition, $\left.\mathrm{NH}\right), 11.20\left(2 \mathrm{H}\right.$, s, exchanged by $\mathrm{D}_{2} \mathrm{O}$ addition, $\left.2 \times \mathrm{NH}\right), 7.54(4 \mathrm{H}, \mathrm{bs}, \mathrm{Ar}), 5.01(1 \mathrm{H}, \mathrm{s}$, $\mathrm{CH}), 3.45$ (3H, s, 3-Me), 3.14 (3H, s, 1-Me); ${ }^{13} \mathrm{C}$ NMR (DMSO-d, $\left.75.5 \mathrm{MHz}\right) \delta 169.5$ (C=O carbonyl), $159.1(\mathrm{C}=\mathrm{O}$ carbonyl), 151.1 (C=O carbonyl), 139.6, 133.0, 130.6, 130.1, 129.7, 129.2 and 129.1 (aromatic C), 111.5 and 101.1 (pyrrole C), $46.9(\mathrm{CH}), 31.0$ and $27.8\left(\mathrm{CH}_{3}\right)$. Anal. Calcd for $\mathrm{C}_{18} \mathrm{H}_{14} \mathrm{ClN}_{5} \mathrm{O}_{5}$ : C, 52.00; H, 3.39; N, 16.84. Found: C, 51.92; H, 3.45; N, 16.90 .

5-(6-(4-Nitrophenyl)-1,3-dimethyl-2,4-dioxo-2,3,4,7-tetrahydro-1H-pyrrolo[2,3-d]pyrimidin-5-yl)pyrimidine2,4,6(1H,3H,5H)-trione (4d). Orange powder: $324 \mathrm{mg}$; mp $>300{ }^{\circ} \mathrm{C}$; FT-IR (KBr) $v_{\max } 3442,3392,3139,2963,1642$, 1589, 1438, 1328, 1438, 1328, 1218, 1148, 1118, 976, 580, 743, 706, $638 \mathrm{~cm}^{-1} ;{ }^{1} \mathrm{H}$ NMR (DMSO-d, $\left.300 \mathrm{MHz}\right) \delta$ $12.16\left(1 \mathrm{H}, \mathrm{s}\right.$, exchanged by $\mathrm{D}_{2} \mathrm{O}$ addition, $\left.\mathrm{NH}\right), 11.28\left(2 \mathrm{H}, \mathrm{s}\right.$, exchanged by $\mathrm{D}_{2} \mathrm{O}$ addition, $\left.2 \times \mathrm{NH}\right), 8.26(2 \mathrm{H}, \mathrm{d}, J=7.8$ $\mathrm{Hz}, \mathrm{Ar}), 7.74$ (2H, d, $J=7.8 \mathrm{~Hz}, \mathrm{Ar}), 5.17$ (1H, s, CH), 3.44 (3H, s, 3-Me), 3.13 (3H, s, 1-Me); ${ }^{13} \mathrm{C}$ NMR (DMSO-d ${ }_{6}$, $75.5 \mathrm{MHz}) \delta 169.3$ (C=O carbonyl), 159.0 (C=O carbonyl), 151.2 (C=O carbonyl), 140.6, 132.7, 132.3, 129.7, 128.7, 
128.3 and 127.4 (aromatic C), 111.9 and 98.2 (pyrrole C), $47.6(\mathrm{CH}), 31.1$ and $27.8\left(\mathrm{CH}_{3}\right)$. Anal. Calcd for $\mathrm{C}_{18} \mathrm{H}_{14} \mathrm{~N}_{6} \mathrm{O}_{7}$ : C, 50.71; H, 3.31; N, 19.71. Found: C, 50.63; H, 3.40; N, 19.81.

\section{6-(Phenyl)-5-(4,6-dioxo-2-thioxohexahydropyrimidin-5-yl)-1,3-dimethyl-1,7-dihydro-2H-pyrrolo[2,3-}

d]pyrimidine-2,4(3H)-dione (4e). Pale pink powder: $341 \mathrm{mg} ; \mathrm{mp}>300{ }^{\circ} \mathrm{C}$ (Lit.[24] $>350^{\circ} \mathrm{C}$ ); FT-IR (KBr) $v_{\max } 3354$, 3178, 2257, 1648, 1550, 1439, 1300, 1219, 1154, 1006, 761, 696, $539 \mathrm{~cm}^{-1}$; ${ }^{1} \mathrm{H}$ NMR (DMSO-d, $\left.300 \mathrm{MHz}\right) \delta 12.29$ $\left(1 \mathrm{H}\right.$, s, exchanged by $\mathrm{D}_{2} \mathrm{O}$ addition, $\left.\mathrm{NH}\right), 11.94\left(1 \mathrm{H}\right.$, s, exchanged by $\mathrm{D}_{2} \mathrm{O}$ addition, $\left.\mathrm{NH}\right), 11.66(1 \mathrm{H}$, s, exchanged by $\mathrm{D}_{2} \mathrm{O}$ addition, $\left.\mathrm{NH}\right), 7.41(2 \mathrm{H}, \mathrm{d}, J=7.2 \mathrm{~Hz}, \mathrm{Ar}), 7.26(2 \mathrm{H}, \mathrm{t}, J=6.9 \mathrm{~Hz}, \mathrm{Ar}), 7.16(1 \mathrm{H}, \mathrm{d}, J=6.6 \mathrm{~Hz}, \mathrm{Ar}), 5.20(1 \mathrm{H}, \mathrm{s}$, $\mathrm{CH}), 3.44$ (3H, s, 3-Me), 3.15 (3H, s, 1-Me); ${ }^{13} \mathrm{C}$ NMR (DMSO-d, $\left.75.5 \mathrm{MHz}\right) \delta 173.8$ (C=S), 161.3 (C=O carbonyl), 158.4 (C=O carbonyl), 151.9 (C=O carbonyl), 139.8, 132.1, 129.7, 129.5 and 129.2 (aromatic C), 106.8 and 100.2 (pyrrole C), $48.9(\mathrm{CH}), 30.9$ and $27.8\left(\mathrm{CH}_{3}\right)$. Anal. Calcd for $\mathrm{C}_{18} \mathrm{H}_{15} \mathrm{~N}_{5} \mathrm{O}_{4} \mathrm{~S}$ : C, 54.40; H, 3.80; N, 17.62. Found: C, 54.49; H, 3.71; N, 17.70 .

6-(Methylphenyl)-5-(4,6-dioxo-2-thioxohexahydropyrimidin-5-yl)-1,3-dimethyl-1,7-dihydro-2H-pyrrolo[2,3d]pyrimidine-2,4(3H)-dione (4f). Pale pink powder: $370 \mathrm{mg}$; mp $>300{ }^{\circ} \mathrm{C}$; FT-IR (KBr) $v_{\max } 3399,3183,3057,2923$, 2854, 1692, 1650, 1554, 1435, 1360, 1237, 1157, 1040, 1005, 875, 763, 743, 629, $534 \mathrm{~cm}^{-1}$; ${ }^{1} \mathrm{H}$ NMR (DMSO-d $\mathrm{d}_{6}$, 300 $\mathrm{MHz}) \delta 12.28$ (s, $1 \mathrm{H}$, exchanged by $\mathrm{D}_{2} \mathrm{O}$ addition, $\left.\mathrm{NH}\right), 11.75\left(1 \mathrm{H}, \mathrm{s}\right.$, exchanged by $\mathrm{D}_{2} \mathrm{O}$ addition, $\left.\mathrm{NH}\right), 11.61(1 \mathrm{H}, \mathrm{s}$, exchanged by $\mathrm{D}_{2} \mathrm{O}$ addition, $\left.\mathrm{NH}\right), 7.21$ (2H, bs, Ar), 6.96 (2H, bs, Ar), $4.66(1 \mathrm{H}, \mathrm{s}, \mathrm{CH}), 3.36$ (3H, s, 3-Me), 3.15 (3H, s, 1-Me), 2.31 (3H, s, Me); ${ }^{13} \mathrm{C}$ NMR (DMSO-d 6 , $\left.75.5 \mathrm{MHz}\right) \delta 173.6(\mathrm{C}=\mathrm{O}), 161.6(\mathrm{C}=\mathrm{O}$ carbonyl), $158.3(\mathrm{C}=\mathrm{O}$ carbonyl), 151.0 (C=O carbonyl), 139.7, 132.2, 132.1, 129.6, 129.4 and 128.9 (aromatic C), 107.0 and 98.3 (pyrrole C), $49.0(\mathrm{CH})$, 30.8, 29.7 and $23.5\left(\mathrm{CH}_{3}\right)$. Anal. Calcd for $\mathrm{C}_{19} \mathrm{H}_{17} \mathrm{~N}_{5} \mathrm{O}_{4} \mathrm{~S}$ : C, 55.47; H, 4.16; N, 17.02. Found: C, 55.40; H, 4.26; N, 17.10 .

6-(4-Chlorophenyl)-5-(4,6-dioxo-2-thioxohexahydropyrimidin-5-yl)-1,3-dimethyl-1,7-dihydro-2H-pyrrolo[2,3d]pyrimidine-2,4(3H)-dione (4g). Pale pink powder: $328 \mathrm{mg}$; mp >300 ${ }^{\circ} \mathrm{C}$; FT-IR (KBr) $v_{\max } 3359$, 3195, 2959, 1648, 1549, 1433, 1378, 1296, 1221, 1152, 1006, 978, 787, 738, 668, $539 \mathrm{~cm}^{-1} ;{ }^{1} \mathrm{H}$ NMR (DMSO-d, $\left.300 \mathrm{MHz}\right) \delta 12.29(1 \mathrm{H}$, s, exchanged by $\mathrm{D}_{2} \mathrm{O}$ addition, $\left.\mathrm{NH}\right), 12.06\left(1 \mathrm{H}, \mathrm{s}\right.$, exchanged by $\mathrm{D}_{2} \mathrm{O}$ addition, $\left.\mathrm{NH}\right), 11.72\left(1 \mathrm{H}\right.$, s, exchanged by $\mathrm{D}_{2} \mathrm{O}$ addition, NH), $7.43(2 \mathrm{H}, \mathrm{d}, J=7.8 \mathrm{~Hz}, \mathrm{Ar}), 7.39(2 \mathrm{H}, \mathrm{d}, J=7.8 \mathrm{~Hz}, \mathrm{Ar}), 4.68(1 \mathrm{H}, \mathrm{s}, \mathrm{CH}), 3.45$ (3H, s, 3-Me), 3.16 (3H, s, 1-Me); ${ }^{13} \mathrm{C}$ NMR (DMSO-d, $\left.75.5 \mathrm{MHz}\right) \delta 174.0$ (C=S), 161.9 (C=O carbonyl), 158.3 (C=O carbonyl), 152.0 (C=O carbonyl), 140.2, 131.7, 131.0, 128.7, 128.6 and 128.3 (aromatic C), 107.3 and 100.1 (pyrrole C), 49.0 (CH), 29.7 and $27.8\left(\mathrm{CH}_{3}\right)$. Anal. Calcd for $\mathrm{C}_{18} \mathrm{H}_{14} \mathrm{ClN}_{5} \mathrm{O}_{4} \mathrm{~S}$ : C, 50.06; H, 3.27; N, 16.22. Found: C, 50.13; H, 3.35; N, 16.13.

6-(4-Nitrophenyl)-5-(4,6-dioxo-2-thioxohexahydropyrimidin-5-yl)-1,3-dimethyl-1,7-dihydro-2H-pyrrolo[2,3d]pyrimidine-2,4(3H)-dione (4h). Orange powder: $323 \mathrm{mg}$; mp $>300{ }^{\circ} \mathrm{C}$; FT-IR (KBr) $v_{\max } 3400,3209,2955,1702$, 1689, 1615, 1495, 1364, 1221, 1095, 827, 744, $514 \mathrm{~cm}^{-1}$; ${ }^{1} \mathrm{H}$ NMR (DMSO-d, $\left.300 \mathrm{MHz}\right) \delta 12.21$ (2H, s, exchanged by $\mathrm{D}_{2} \mathrm{O}$ addition, $\left.2 \times \mathrm{NH}\right), 11.86\left(1 \mathrm{H}, \mathrm{s}\right.$, exchanged by $\mathrm{D}_{2} \mathrm{O}$ addition, $\left.\mathrm{NH}\right), 8.10(2 \mathrm{H}, \mathrm{d}, J=7.8 \mathrm{~Hz}, \mathrm{Ar}), 7.62(2 \mathrm{H}, \mathrm{d}, J=7.8$ $\mathrm{Hz}, \mathrm{Ar}), 4.55$ (1H, s, CH), 3.42 (3H, s, 3-Me), 3.14 (3H, s, 1-Me); ${ }^{13} \mathrm{C}$ NMR (DMSO-d, $\left.75.5 \mathrm{MHz}\right) \delta 174.1$ (C=S), 161.0 ( $\mathrm{C}=\mathrm{O}$ carbonyl), 158.2 ( $\mathrm{C}=\mathrm{O}$ carbonyl), 150.9 (C=O carbonyl), 141.1, 131.6, 127.5, 126.5, 125.0 and 122.8 (aromatic C), 110.4 and 100.9 (pyrrole C), $48.1(\mathrm{CH}), 31.0$ and $27.8\left(\mathrm{CH}_{3}\right)$. Anal. Calcd for $\mathrm{C}_{18} \mathrm{H}_{14} \mathrm{~N}_{6} \mathrm{O}_{6} \mathrm{~S}: \mathrm{C}, 48.98 ; \mathrm{H}$, 3.08; N, 19.11. Found: C, 48.87; H, 3.19; N, 19.00.

5-(6-(Phenyl)-1,3-dimethyl-2,4-dioxo-2,3,4,7-tetrahydro-1H-pyrrolo[2,3-d]pyrimidin-5-yl)-1,3dimethylpyrimidine-2,4,6(1H,3H,5H)-trione (4i). White powder: $372 \mathrm{mg}$; mp $>300{ }^{\circ} \mathrm{C}$ (Lit.[24] >350 ${ }^{\circ} \mathrm{C}$ ); FT-IR $(\mathrm{KBr}) v_{\max } 3450,3177,2963,1691,1640,1565,1495,1372,1248,1091,1053,975,819,742,517 \mathrm{~cm}^{-1} ;{ }^{1} \mathrm{H} \mathrm{NMR}$ (DMSO-d $6,300 \mathrm{MHz}) \delta 11.90$ (1H, s, exchanged by $\mathrm{D}_{2} \mathrm{O}$ addition, NH), 7.35-7.65 (5H, m, Ar), 5.15 (1H, s, CH), 3.50 (3H, s, Me), 3.16 (6H, s, 2xMe), 3.13 (3H, s, Me); ${ }^{13} \mathrm{C}$ NMR (DMSO-d, $\left.75.5 \mathrm{MHz}\right) \delta 168.1$ (C=O carbonyl), 159.3 (C=O carbonyl), 152.4(C=O carbonyl), 151.1 (C=O carbonyl), 139.7, 131.6, 130.8, 129.6 and 128.5 (aromatic C), 109.2 and 97.3 (pyrrole C), $47.1(\mathrm{CH}), 31.1,29.3$ and $28.9\left(\mathrm{CH}_{3}\right)$. Anal. Calcd for $\mathrm{C}_{20} \mathrm{H}_{19} \mathrm{~N}_{5} \mathrm{O}_{5}$ : C, 58.68; H, 4.68; N, 17.11. Found: C, 58.60; H, 4.77; N, 17.19 .

\section{5-(6-(4-Methylphenyl)-1,3-dimethyl-2,4-dioxo-2,3,4,7-tetrahydro-1H-pyrrolo[2,3-d]pyrimidin-5-yl)-1,3-}

dimethylpyrimidine-2,4,6(1H,3H,5H)-trione (4j). White powder: $398 \mathrm{mg}$; mp > 300 C; FT-IR (KBr) $v_{\max } 3745$, 3459, 2938, 1691, 1634, 1446, 1344, 1215, 1107, 1033, 905, 760, $631 \mathrm{~cm}^{-1}$; ${ }^{1} \mathrm{H}$ NMR (DMSO- $\left.d_{6}, 300 \mathrm{MHz}\right) \delta 11.85(1 \mathrm{H}, \mathrm{s}$, exchanged by $\mathrm{D}_{2} \mathrm{O}$ addition, $\left.\mathrm{NH}\right), 7.38(2 \mathrm{H}, \mathrm{d}, J=7.5 \mathrm{~Hz}, \mathrm{Ar}), 7.13(2 \mathrm{H}, \mathrm{d}, J=7.5 \mathrm{~Hz}, \mathrm{Ar}), 5.16(1 \mathrm{H}, \mathrm{s}, \mathrm{CH}), 3.52(3 \mathrm{H}$, s, 3-Me), 3.33 (3H, s, 1-Me), 3.05 (6H, s, 1-Me, 3-Me), 2.26 (3H, s, Me); ${ }^{13} \mathrm{C}$ NMR (DMSO-d, $\left.75.5 \mathrm{MHz}\right) \delta 167.8$ (C=O carbonyl), 159.2 ( $\mathrm{C}=\mathrm{O}$ carbonyl), 152.3 (C=O carbonyl), 151.0 ( $\mathrm{C}=\mathrm{O}$ carbonyl), 140.7, 132.1, 129.7129 .3 and 129.3 (aromatic C), 112.1 and 100.6 (pyrrole C), $47.7(\mathrm{CH}), 31.2,28.9,27.9$ and $21.2\left(\mathrm{CH}_{3}\right)$. Anal. Calcd for $\mathrm{C}_{21} \mathrm{H}_{21} \mathrm{~N}_{5} \mathrm{O}_{5}$ : C, 59.57; H, 5.00; N, 16.54. Found: C, 59.45; H, 5.00; N, 16.62. 
5-(6-(4-Chlorophenyl)-1,3-dimethyl-2,4-dioxo-2,3,4,7-tetrahydro-1H-pyrrolo[2,3-d]pyrimidin-5-yl)-1,3dimethylpyrimidine-2,4,6(1H,3H,5H)-trione (4k). Pale yellow powder: $364 \mathrm{mg}$; mp > $300{ }^{\circ} \mathrm{C}$; FT-IR $(\mathrm{KBr}) v_{\max } 3392$, 3220, 3108, 2951, 2875, 1703, 1651, 1562, 1443, 1359, 1241, 1038, 975, 816, 774, 702, 608, $502 \mathrm{~cm}^{-1} ;{ }^{1} \mathrm{H}$ NMR $\left(\mathrm{DMSO}_{6}, 300 \mathrm{MHz}\right) \delta 11.95\left(1 \mathrm{H}, \mathrm{s}\right.$, exchanged by $\mathrm{D}_{2} \mathrm{O}$ addition, $\left.\mathrm{NH}\right), 7.57$ (4H, bs, Ar), $5.16(1 \mathrm{H}, \mathrm{s}, \mathrm{CH}), 3.49(3 \mathrm{H}$, s, 3-Me), 3.16 (6H, s, 1-Me, 3-Me), 2.50 (3H, s, Me); ${ }^{13} \mathrm{C}$ NMR: (DMSO-d $\left.d_{6}, 75.5 \mathrm{MHz}\right) \delta 168.0$ (C=O carbonyl), 159.2 (C=O carbonyl), 152.4 (C=O carbonyl), 139.9, 133.1, 130.4, 130.1, 129.6 and 129.3 (aromatic C), 109.7 and 101.0 (pyrrole C), $47.2(\mathrm{CH}), 31.1,30.4$ and $28.9\left(\mathrm{CH}_{3}\right)$. Anal. Calcd for $\mathrm{C}_{20} \mathrm{H}_{18} \mathrm{ClN}_{5} \mathrm{O}_{5}$ : C, 54.12; H, 4.09; N, 15.78. Found: C, 54.20; H, 4.16; N, 15.70 .

\section{5-(6-(4-Nitrophenyl)-1,3-dimethyl-2,4-dioxo-2,3,4,7-tetrahydro-1H-pyrrolo[2,3-d]pyrimidin-5-yl)-1,3-} dimethylpyrimidine-2,4,6(1H,3H,5H)-trione (4l). Orange powder: $341 \mathrm{mg}$; mp >300 ${ }^{\circ} \mathrm{C}$; FT-IR $(\mathrm{KBr}) v_{\max } 3178$, 3069, 2940, 1700, 1670, 1581, 1514, 1444, 1341, 1109, 979, 860, 752, $706 \mathrm{~cm}^{-1} ;{ }^{1} \mathrm{H}$ NMR (DMSO-d, $\left.300 \mathrm{MHz}\right) \delta$ 12.15 (1H, s, exchanged by $\mathrm{D}_{2} \mathrm{O}$ addition, $\left.\mathrm{NH}\right), 8.34$ (2H, d, $\left.J=8.4 \mathrm{~Hz}, \mathrm{Ar}\right), 7.80(2 \mathrm{H}, \mathrm{d}, J=8.4 \mathrm{~Hz}, \mathrm{Ar}), 5.32(1 \mathrm{H}, \mathrm{s}$, $\mathrm{CH}), 3.51$ (3H, s, 3-Me), 3.15 (6H, s, 1-Me, 3-Me), 2.50 (3H, s, Me); ${ }^{13} \mathrm{C}$ NMR: (DMSO-d, $\left.75.5 \mathrm{MHz}\right) \delta 167.8(\mathrm{C}=\mathrm{O}$ carbonyl), 159.2 (C=O carbonyl), 152.3 (C=O carbonyl), 151.0 (C=O carbonyl), 140.7, 132.0, 129.7, 129.6 and 129.3 (aromatic C), 112.1 and 102.7 (pyrrole C), $47.7(\mathrm{CH})$, 31.2, 28.9 and $27.9\left(\mathrm{CH}_{3}\right)$. Anal. Calcd for $\mathrm{C}_{20} \mathrm{H}_{18} \mathrm{~N}_{6} \mathrm{O}_{7}$ : C, 52.87; H, 3.99; N, 18.50. Found: C, 52.73; H, 4.05; N, 18.63.

\section{Acknowledgment}

We are grateful to Urmia University for financial support. We also thank Professor R.H. Prager from Flinders University, Australia for proof-reading and language editing of manuscript.

\section{References}

1. (a) Khodabakhshi, S.; Karimi, B. New J. Chem. 2014, 38, 3586-3590. (b) Bagdi, A.; Hajra, A. RSC Adv. 2014, 4, 23287-23291. (c) Ma, L. Y.; Zheng, Y. C.; Wang, S. Q.; Wang, B.; Wang, Z. R.; Pang, L. P.; Zhang, M.; Wang, J. W.; Ding, L.; Li, J.; Wang C.; Hu, B.; Liu, Y.; Zhang, X. D.; Wang, J. J.; Wang, Z. J.; Zhao, W.; Liu, H. M. J. Med. Chem. 2015, 58, 1705-1716.

2. (a) Mahmudov, K. T.; Kopylovich, M. N.; Maharramov, A. M.; Kurbanova, M. M.; Gurbanov, A. V.; Pombeiro, A. J. L.; Coord. Chem. Rev. 2014, 265, 1-37. (b) Chanda, A.; Fokin, V. V. Chem. Rev. 2009, 109, 725-748. (c) Allias, C.; Grassot, J.; Rodriguez, J.; Constantieux, T. Chem. Rev. 2014, 114, 10829-10868. (d) Domling, A.; Wang, W.; Wang, K. Chem. Rev. 2012, 12, 3083-3135.

3. (a) Jiang, S.; Gao, J.; Han, L. Res. Chem. Intermed. 2016, 2, 1017-1028. (b) Teimuri-Mofrad, R.; Shahrisa, A.; Gholamhosseini-Nazari, M.; Arsalani, N. Res. Chem. Intermed. 2016, 42, 3425-3439. (c) Nikoofar, K.; Khademi, Z. Res. Chem. Intermed. 2016, 42, 3929-3977. (d) Ershov, O. V.; Ievler, M. Y.; Tafeenko, V. A.; Nasakin, O. E. Green Chem. 2015, 17, 4234-4238. (e) Vidadala, S. R.; Waldmann, H. Tetrahedron Lett. 2015, 56, 3358-3360. (f) Peck, C. L.; Calderone, J. A.; Santos, W. L. Synthesis 2015, 47, 2242-2248. (g) Fu, Z.; Qian, K.; Li, S.; Shen, T. Tetrahedron Lett. 2016, 57, 1104-1108.

4. (a) Ma, L.; Li, S.; Zheng, H.; Chen, J.; Lin, L.; Ye, X.; Chen, Zhizhi.; Xu, Q.; Chen, T.; Yang, J.; Qiu, N.; Wang, G.; Peng, A.; Ding, Y.; Wei, Y.; Chen, L. Eur. J. Med. Chem. 2011, 46, 2003-2010. (b) Edupuganti, R.; Wang, Q.; Tavares, C. D. J.; Chitjian, C. A.; Bachman, J. L.; Ren, P.; Anslyn, E. V.; Dalby, K. N. Bioorg. Med. Chem. 2014, 22, 4910-4916. (c) Pałasz, A.; Cież, D. Eur. J. Med. Chem. 2015, 97, 582-611.

5. (a) Tolstoluzhsky, N.; Nikolaienko, P.; Gorobets, N.; Van der Eycken, E. V.; Kolos, N. Eur. J. Org. Chem. 2013, 2013, 5364-5369. (b) Sisa, M.; Pla, D.; Altuna, M.; Francesch, A.; Cuevas, C.; Albericio, F.; Alvarez, M. J. Med. Chem. 2009, 52, 6217-6223. (c) Giraud, F.; Alves, G.; Debiton, E.; Nauton, L.; Thery, V.; Durieu, E.; Ferandin, Y.; Lozach, O.; Meijer, L.; Anizon, F.; Pereira, E.; Moreau, P. J. Med. Chem. 2011, 54, 4474-4489.

6. Dhorajiya, B. D.; Dholakiya, B. Z.; Mohareb, R. M. Med. Chem. Res. 2014, 23, 3941-3952.

7. Yagi, K.; Akimoto, K.; Mimori, N.; Miyake, T.; Kudo, M.; Arai, K.; Ishii, S. Pest Manage. Sci. 2000, 56, 65-73.

8. (a) Isobe, Y.; Tobe, M.; Inoue, Y.; Isobe, M.; Tsuchiya, M.; Hayashi, H. Bioorg. Med. Chem. 2003, 11, 49334940. (b) El-Gazzar, A. R. B. A.; Hafez, H. N. Bioorg. Med. Chem. Lett. 2009, 19, 3392-3397.

9. (a) Siddiqui, Z. N. Tetrahedron Lett. 2015, 56, 1919-1924. (b) Singh, P.; Kaur, M.; Verma, P. Bioorg. Med. Chem. Lett. 2009, 19, 3054-3058. (c) Dhorajiya, B. D.; Dholakiya, B. Z.; Mohareb, R. M. Med. Chem. Res. 2014, 23, 3941-3952.

10. Abu-Hashem, A. A.; Gouda, M. A.; Badria, F. A. Eur. J. Med. Chem. 2010, 45, 1976-1981.

11. Kliethermes, C. L.; Metten, P.; Belknap, J. K.; Buck, K. J.; Crabbe, J. C. Brain Res. 2004, 1009, 17-25.

12. (a) Archana, V. K.; Srivastava, Kumar, A. Bioorg. Med. Chem. 2004, 12, 1257-1264. (b) Mahmudov, K. T.; Kopylovich, M. N.; Maharramov, A. M.; Kurbanov, M. M. Int. J. Res. Pharm. Biomed. Sci. 2011, 2, 842-845. 
13. Sandberg, F. Acta Physiol. Scand. 1951, 24, 7-26.

14. (a) Starks, C. M.; Liotta, C. L.; Halpern, M. Phase-Transfer Catalysis; Chapman \& Hall: New York, 1994. (b) Handbook of Phase-Transfer Catalysis; Sasson, Y.; Neumann, R. (Eds.); Blackie Academic \& Professional: London, 1997. (c) Phase Transfer Catalysis; Halpern, M. E. (Ed.); ACS Symposium Series 659; American Chemical Society: Washington, DC, 1997. (d) Dehmlow, E. V.; Dehmlow, S. S. Phase Transfer Catalysis; 3rd ed.; VCH: Weinheim, 1993.

15. (a) Kantevari, S.; Chary, M. V.; Das, A. P. R.; Vuppallapati, S. V. N.; Lingaiah, N. Catal. Commun. 2008, 9, 1575-1578. (b) Chary, M. V.; Keerthysri, N. C.; Vapallapati, S. V. N.; Lingaiah, N.; Kantevari, S. Catal. Commun. 2008, 9, 2013-2017. (c) Ronchin, L.; Vavasori, A.; Amadio, E.; Cavinato, G.; Toniolo, L. J. Mol. Catal. A: Chem. 2009, 298, 23-30.

16. Siddiqui, S. A.; Narkhede, U. C.; Palimkar, S. S.; Daniel, T.; Loholi, R. J.; Srinivasan, K. V. Tetrahedron, 2005, 61, 3539-3546. (b) Amantini, D.; Fringuelli, F.; Pizzo, F.; Vaccaro, L. J. Org. Chem. 2001, 66, 6734-6737. (c) Khurana, J. M.; Kumar, S. Tetrahedron Lett. 2009, 50, 4125-4127.

17. Khalafy, J.; Majidi Arlan, F.; Soleimani Chalanchi, Sh. J. Heterocycl. Chem. 2018, 55, 149-153.

18. Ezzati, M.; Khalafy, J.; Poursattar Marjani, A.; Prager, R. H. Tetrahedron 2017, 73, 6587-6596.

19. Poursattar Marjani, A.; Khalafy, J.; Rostampoor, A. J. Heterocycl. Chem. 2017, 54, 648-652.

20. Khalafy, J.; Badparvar, F.; Poursattar Marjani, A. J. Chil. Chem. Soc. 2016, 61, 3112-3115.

21. Poursattar Marjani, A.; Khalafy, J.; Chitan, M.; Mahmoodi, S. Iran. J. Chem. Chem. Eng. 2017, 36, 1-6.

22. Javahershenas, R.; Khalafy, J. J. Heterocycl. Chem. 2017, 54, 3163-3168.

23. Javahershenas, R.; Khalafy, J. Heterocycl Commun. 2018, 24(1), 37-42.

24. Dommaraju, Y.; Borthakur, S.; Rajesh, N.; Prajapati, D. RSC Adv. 2015, 5, 24327-24335.

25. Perrin, D. D.; Armarego, W. L. F. Purification of Laboratory Chemicals, Pergamon Press: Oxford, U.K., 1988. 